$\xi=-1$ 国

\title{
Premature Ventricular Contraction Classification based on ECG Signal using Multilevel Wavelet entropy
}

\author{
Achmad Rizal $^{1 *}$, Riandini' $^{2}$, Teni Tresnawati ${ }^{3}$ \\ ${ }^{1}$ School of Electrical engineering, Telkom University, Indonesia \\ ${ }^{2}$ Politeknik Negeri Jakarta, Indonesia \\ ${ }^{3}$ Politeknik Negeri Jakarta, Indonesia \\ *Corresponding author E-mail: achmadrizal@telkomuniversity.ac.id
}

\begin{abstract}
One of the abnormalities in the heart that can be assessed from an ECG signal is premature ventricle contraction (PVC). PVC is a form of arrhythmia in the form of irregularity in beat ECG signals. In this study, a multilevel wavelet entropy method was developed to distinguish PVC and normal ECG signals automatically. Data was taken from the MIT-BIH arrhythmia database with the process carried out is normalization, median filtering, beat-parsing, MWE calculation and classification using SVM. The results of the experiment showed that MWE level 5 with DB2 as mother wavelet and Quadratic SVM as classifier resulted in the highest accuracy of $94.9 \%$. MWE level 5 means only five features needed for classification. The number of features is very little compared to previous research with a quite high accuracy.
\end{abstract}

Keywords: Premature ventricle contraction; electrocardiogram; multilevel wavelet entropy; support vector machine; arrhythmia

\section{Introduction}

The Health of someone's heart could be seen from the ECG signal generated from heart activities. ECG signals are rated by rhythm, shape, and orientation [1]. Shape change, rhythm, and ECG signal orientation indicate the existence of heart abnormalities. One of the heart abnormalities is an arrhythmia. Arrhythmia is caused by heartbeat irregularity, speed interference, or problem in heart electricity signal transmission [2]. One of arrhythmias type is premature ventricle contraction (PVC). PVC is caused by early myocardial depolarization originating from the ventricular region. Lots of PVC happens in adults.

The various method has been developed to detect PVC or other arrhythmia using a digital signal processing technique. Lanata et al. used the bispectral method for classification of five types of arrhythmia [3]. While, Mitra and Samanta used correlation-based feature subset selection (CFS), principal component analysis (PCA), association rules (AR), and rough set theory to reduce ECG signal dimensions [4]. Kaya and Pehlivan used PCA, ICA, and SOM for data reduction on PVC ECG signal. Neural network (NN), support vector machine (SVM), K-nearest neighbor $(\mathrm{KNN})$, and decision tree (DT) were used as classifiers [5]. Previous researches chose to use feature extraction technique while some only used signal dimensions reduction method.

In this study, the entropy wavelet-based method called the multilevel wavelet entropy (MWE) was used for PVC classification. Wavelet entropy was proposed by Rosso et al. for EEG signal analysis [6]. Usually, WE was only calculated on one decomposition level, but on this research, WE was calculated on several decomposition levels, so it is called multilevel wavelet entropy (MWE). WE measurement on several decomposition levels is expected to give more complete information about ECG signal energy distribution.
The proposed method merged with SVM was expected to give high accuracy for PVC classification using ECG signal.

\section{Material and Methods}

Figure 1 shows the block diagram of this study. At first, the original ECG signal was preprocessed for normalization and noise reduction Then the parsing beat process is performed to break the ECG signal into one cycle of ECG signals. Next feature extraction was done in the form of entropy wavelet calculations at several levels. Entropy wavelets will be used as input for the classification process. The results obtained are classification accuracy. The more detailed explanation is given in the following subsection.

\subsection{ECG Database}

The ECG signal data used in this study came from The MIT-BIH Arrhythmia Database [7]. Data were selected which have normal ECG and PVC signals. Each data was taken with a sampling frequency of $360 \mathrm{~Hz}$ from lead II and one additional lead. Each data has an annotation that shows the time and beat label in the form of an $\mathrm{R}$ wave location. In this study, ten files were used with the number of each normal ECG wave and PVC as in Table 1.

\subsection{Pre-processing}

Preprocessing is intended to reduce the noise that occurs in the signal. There are two processes performed on the input signal. First is the average reduction as in Equation (1) [8].

$s(t)=x(t)-\bar{x}$ 


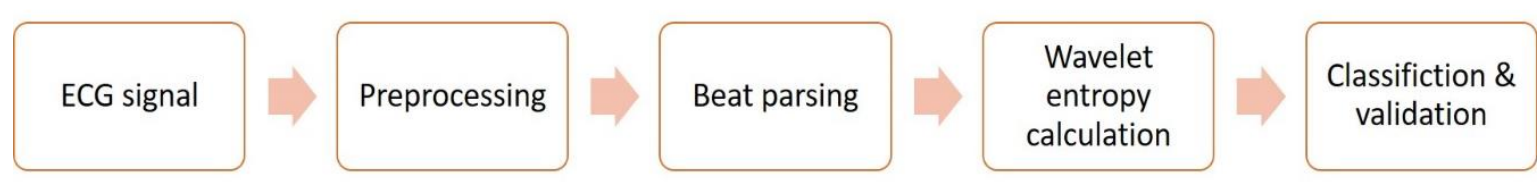

Fig. 1: Diagram block of system

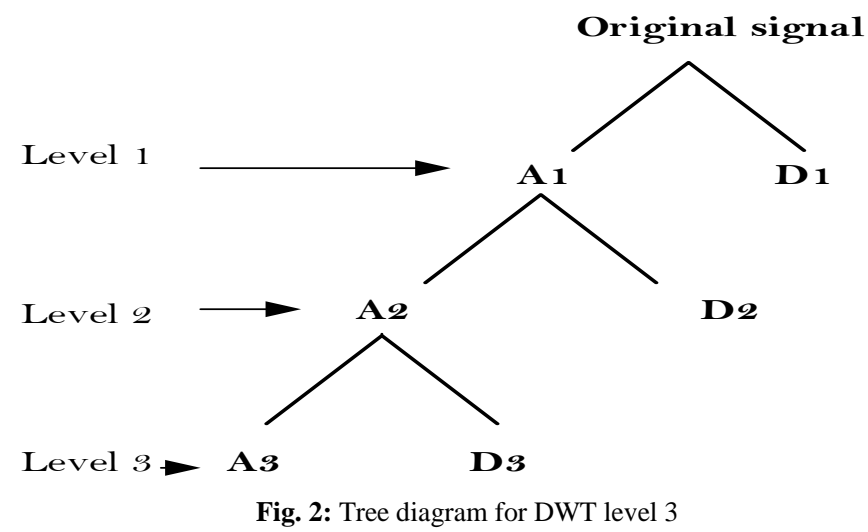

Table 1: Total number of beat from selected files in MIT-BIH database

\begin{tabular}{|c|c|c|c|}
\hline Filename & Normal & PVC & Total \\
\hline 106 & 0 & 520 & 520 \\
\hline 108 & 1738 & 17 & 1755 \\
\hline 109 & 0 & 38 & 38 \\
\hline 114 & 1820 & 43 & 1863 \\
\hline 116 & 0 & 109 & 109 \\
\hline 118 & 0 & 16 & 16 \\
\hline 119 & 1543 & 444 & 1987 \\
\hline 124 & 0 & 47 & 47 \\
\hline 200 & 0 & 826 & 826 \\
\hline 201 & 1625 & 198 & 1823 \\
\hline Total & 6726 & 2258 & 8984 \\
\hline
\end{tabular}

where $x(t)$ is the input signal, $\bar{x}$ is the average of the input signal, and $s(t)$ is the output signal.

In the next process, baseline wander removal using a median filter is carried out. Median signals are selected from three consecutive signal samples to eliminate fluctuations that occur in the signal This filtering process is expressed in Equation (2) [5]

$y(t)=\operatorname{med}(s(t-1), s(t), s(t+1))$

where $y(t)$ is the output signal, $s(t)$ is the input signal. The result of this process was ECG signals that were more even and not volatile.

\subsection{Beat parsing}

Beat parsing was done to cut the ECG signal data into one cycle of ECG signals. One QRS pattern in each cycle indicated a cycle of the ECG signal. Because there has been an annotation of the R signal on the long signal data one ECG signal was calculated from the $\mathrm{R}$ signal. One ECG signal is 200 samples in length then one ECG signal is determined as equation (3)

$E C G_{i}=\left[y\left(n_{i}-99\right), \ldots, y\left(n_{i}\right), \ldots, y\left(n_{i}+100\right)\right]$

where $E C G_{i}$ is the $i^{\text {th }}$ ECG signal, $y(n)$ is the input signal and $n_{i}$ is the sample signal which is the $i^{\text {th }} \mathrm{R}$ signal.

\subsection{Multilevel wavelet entropy}

Discrete wavelet transform $(D W T)$ for any signal $s(t)$ can be expressed by Equation (4) [9]:

$\left(W_{\psi} S\right)(j, k)=\int_{-\infty}^{+\infty} S(t) \psi_{j, k}(t) d t$ where $\psi_{j, k}(t)$ is discrete mother wavelet function, $j$ and $k$ are scale and translation parameters, and $j \neq 0$. The tree diagram for the $3 \mathrm{rd}$ order DWT is given in Figure 2.

If given the wavelet coefficient as follows $C_{j}(k)=\left\langle S, \psi_{j, k}\right\rangle$ which is the result of DWT, then the energy of the signal is on a scale $j=$ $1,2, \ldots, N$ is stated as Equation (5).

$E_{j}=\sum_{k}\left|C_{j}(k)\right|^{2}$

The total energy of the DWT signal can be expressed by Equation (6).

$E_{\text {tot }}=\|S\|^{2}=\sum_{j} \sum_{k}\left|C_{j}(k)\right|^{2}=\sum_{j} E_{j}$

The relative energy of wavelets for scale $j$ is expressed by Equation (7)

$P_{j}=\frac{E_{j}}{E_{t o t}}$

So wavelet entropy (WE) can be defined as Equation (8) [6].

$W E=-\sum p_{i} \ln p_{i}$

In this study multilevel wavelet entropy (MWE) is proposed. If the DWT is $\mathrm{N}$-order, MWE will generate $\mathrm{N}$ features in the form of WE in order 1 to N. So that it can be written as Equation (9).

$M W E_{N}=\left[W E_{1}, W E_{2}, \ldots, W E_{N}\right]$

with a $\mathrm{WE}_{1}$ value of zero that can be used or ignored. In this study, the value of $N=5$, and $W_{1}$ is used as a feature. So the features produced by MWE are 5 . 


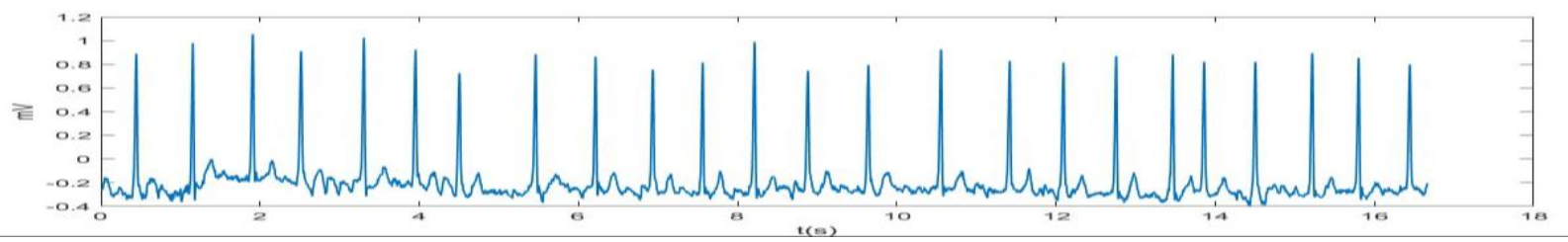

(a)

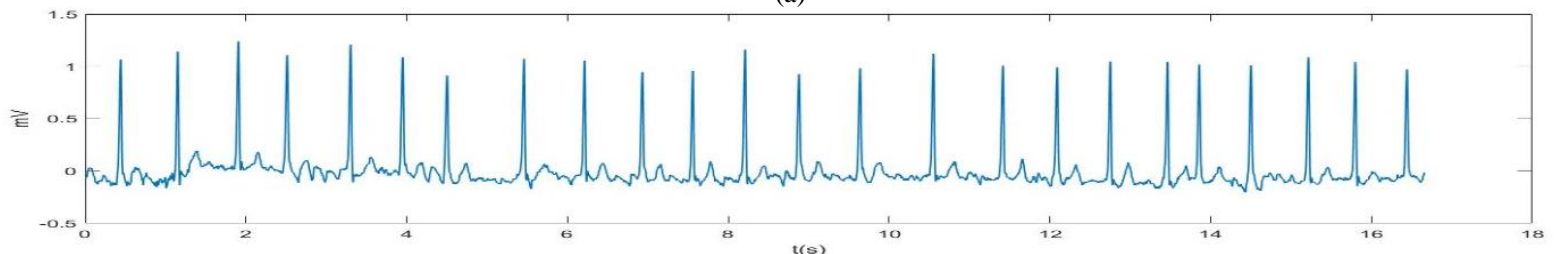

(b)

Fig. 3: (a) original signal (b) signal after preprocessing

\subsection{Classification and validation}

This study used the support vector machine (SVM) as a classifier. SVM is a classifier that uses a hyperplane to separate one class from another class [10]. The characteristics that become input from SVM were transformed into high dimensional feature space and further separated by hyperplane. The hyperplane is a straight line or field that separates data between classes. The best hyperplane was obtained by maximizing the margin between two objects from different classes. The margin was the distance between the hyperplane with the closest pattern in each data class. The closest position between the patterns of each class was called support vector. In a condition where linear or linear lines could not separate characteristic, a method called kernel trick was needed. In addition to being used linearly, SVM also used quadratic SVM and Cubic SVM.

SVM requires a training process before it was used for the classification process. For the distribution of training data and test data, Nfold cross-validation (N-Fold CV) was used. In this research $\mathrm{N}=5$ and $\mathrm{N}=10$ is defined. At $\mathrm{N}=5$, the data was divided into five datasets with one data set as test data and four data sets as training data. This process is repeated five times until all datasets have become test data. This method was better regarding more stable accuracy compared to the random distribution of training data and testing data [11].

\section{Results and Discussion}

Figure 3 displays the initial ECG signal and after preprocessing. After preprocessing, the amplitude increases because the average value of the initial signal is negative. Meanwhile, the median filtering reduces noise and baseline shifts from the original signal. Median filtering uses three consecutive signal samples as input. The use of more signal samples can be tried to get a more flat signal. Feature extraction begins with the wavelet decomposition process using several levels. Like Figure 2, at level 1 generated A1 and D1 subband with bandwidth $0-90 \mathrm{~Hz}$ and $90-180 \mathrm{~Hz}$. Next, at level 2 , subband A2, D2, and D1 were produced with a bandwidth of 0-45 $\mathrm{Hz}, 45-90 \mathrm{~Hz}$, and $90-180 \mathrm{~Hz}$. So on so that at level 5, six subbands were produced, namely A5, D5, D4, ..., and D1. WE was calculated at each decomposition level so that it produces one WE value at each decomposition level. The MWE calculation results for some mother wavelets are shown in Figure 4. The WE value at the low decomposition level occurs because the signal energy is relatively concentrated at low frequencies $(<50 \mathrm{~Hz})$. This causes the energy subband D1 to be much lower than the subband A1 energy. Uneven energy distribution like this makes the entropy value low [12]. It appears that the MWE PVC value is higher than the normal ECG, this is influenced by the nature of PVC which is much more volatile than the normal ECG. Signals with higher fluctuations have higher

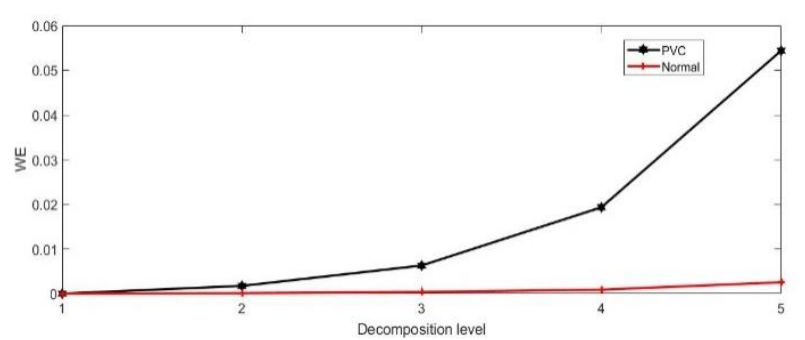

(a)

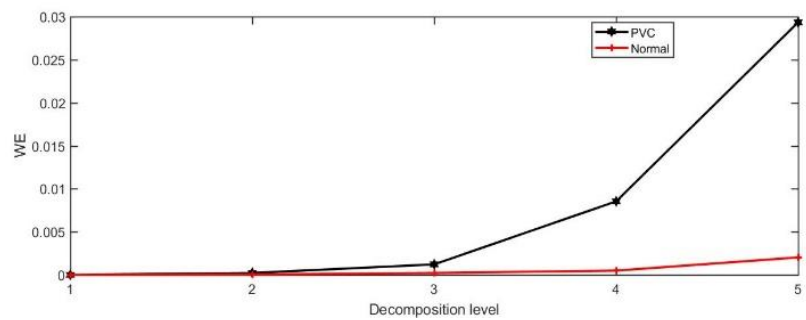

(b)

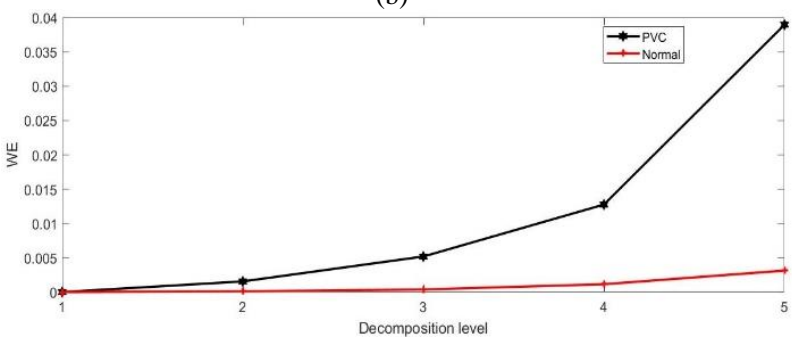

(c)

Fig. 4: Plot of MWE for $\mathrm{N}=5$ (a) Haar (b) using Db2 (c) Bior2.4

complexity resulting in higher entropy. Figure 4 does not show a significant difference between MWE values using different mother wavelets. The selection of mother wavelet affects the order of the wavelet filters associated with filter response. Meanwhile, on WE only calculated the energy probability relative to each subband. Visually the effect of selecting mother wavelet does not affect the pattern of the resulting characteristics, but it adheres to the accuracy obtained.

Table 2 and Table 3 presents the classification accuracy using three kinds of SVM, two types of $\mathrm{N}$-fold $\mathrm{CV}$, five types of mother wavelet, and four types of decomposition level. The highest accuracy is generated by Quadratic SVM with Db2 mother wavelet, 5 fold CV, and level decomposition of 5 which is $94.9 \%$. On average, Quadratic SVM produces higher accuracy for higher decomposition level compared to other SVM. But, if decomposition level was reduced that mean the number of features was reduced, the accuracy of quadrature SVM decrease quickly. 
Table 2: Accuracy using 5-fold CV

\begin{tabular}{|c|c|c|c|c|c|c|c|c|c|c|c|c|c|c|c|}
\hline \multirow{2}{*}{$\begin{array}{c}\text { Decomposi- } \\
\text { tion level }\end{array}$} & \multicolumn{3}{|c|}{ Haar } & \multicolumn{3}{|c|}{$\mathrm{Db} 2$} & \multicolumn{3}{|c|}{ Db8 } & \multicolumn{3}{|c|}{ Bior1.5 } & \multicolumn{3}{|c|}{ Bior2.4 } \\
\hline & $\mathrm{L}$ & $\mathrm{Q}$ & $\mathrm{C}$ & $\mathrm{L}$ & $\mathrm{Q}$ & $\mathrm{C}$ & $\mathrm{L}$ & $\mathrm{Q}$ & $\mathrm{C}$ & $\mathrm{L}$ & $\mathrm{Q}$ & $\mathrm{C}$ & $\mathrm{L}$ & $\mathrm{Q}$ & $\mathrm{C}$ \\
\hline 2 & 50.9 & 33.9 & 62.2 & 54.5 & 39.5 & 64.7 & 74.9 & 38.1 & 64.9 & 55.5 & 31.8 & 74.8 & 39.3 & 38.4 & 68.3 \\
\hline 3 & 74.9 & 36.7 & 57.2 & 74.9 & 48.1 & 71.2 & 74.9 & 46.5 & 64.8 & 74.9 & 42.6 & 54.7 & 74.9 & 37.7 & 70.3 \\
\hline 4 & 91.6 & 89.3 & 76.1 & 74.9 & 58.2 & 45.2 & 74.9 & 58 & 54.6 & 82.3 & 87.3 & 58.7 & 82.2 & 86.6 & 64.6 \\
\hline 5 & 92.5 & 94.1 & 80.6 & 85.8 & 94.9 & 56.8 & 92.5 & 94.2 & 65.7 & 88.6 & 92.9 & 68.3 & 92.5 & 94.1 & 70 \\
\hline
\end{tabular}

$\mathrm{L}=$ linear SVM, $\mathrm{Q}=$ quadrature $\mathrm{SVM}, \mathrm{C}=$ cubic $\mathrm{SVM}$

Table 3: Accuracy using 10-fold CV

\begin{tabular}{|c|c|c|c|c|c|c|c|c|c|c|c|c|c|c|c|}
\hline \multirow{2}{*}{$\begin{array}{c}\text { Decomposition } \\
\text { level }\end{array}$} & \multicolumn{3}{|c|}{ Haar } & \multicolumn{3}{|c|}{ Db2 } & \multicolumn{3}{|c|}{ Db8 } & \multicolumn{3}{|c|}{ Bior1.5 } & \multicolumn{3}{|c|}{ Bior2.4 } \\
\hline & $\mathrm{L}$ & $Q$ & $\mathrm{C}$ & $\mathrm{L}$ & $\mathrm{Q}$ & $\mathrm{C}$ & $\mathrm{L}$ & $Q$ & $\mathrm{C}$ & $\mathrm{L}$ & Q & $\mathrm{C}$ & $\mathrm{L}$ & $\mathrm{Q}$ & $\mathrm{C}$ \\
\hline 2 & 53.1 & 31.4 & 59.7 & 66.1 & 48 & 62.3 & 64.9 & 44.1 & 49.9 & 44.6 & 31.2 & 71.2 & 47.3 & 36.1 & 36.1 \\
\hline 3 & 74.9 & 41.3 & 52.7 & 74.9 & 55.1 & 72 & 74.9 & 48.1 & 40 & 74.9 & 45.1 & 68.6 & 74.9 & 40.1 & 63.6 \\
\hline 4 & 91.5 & 88.8 & 70.4 & 74.9 & 57.7 & 58.9 & 74.9 & 50.9 & 58.6 & 82.3 & 86.2 & 67.3 & 82.2 & 77.2 & 57.2 \\
\hline 5 & 92.5 & 94.1 & 79.9 & 92.4 & 94.1 & 71.2 & 84.9 & 87.2 & 52 & 88.5 & 92.7 & 65.7 & 92.5 & 94.2 & 71.3 \\
\hline
\end{tabular}

$\mathrm{L}=$ linear SVM, $\mathrm{Q}=$ quadrature SVM, C = cubic SVM

The difference between 5fold CV and 10fold CV was in the number of dataset used as training data and testing data. The 5fold CV produced higher accuracy although theoretically 10 fold $\mathrm{CV}$ make the classifier smarter than 5 fold $\mathrm{CV}$ since more dataset was used as training data.

Lower decomposition level (2, 3, and 4) produced lower accuracy than decomposition level 5. It can be seen in Figure 3 and Figure 4 that WE for decomposition level 5 produced more distinct that WE form lower decomposition level. The selection of decomposition level would affect the width of subband which would also affect the value of WE. Using sampling frequency $360 \mathrm{~Hz}$, decomposition level 1 would produce subband A1 $(0-90 \mathrm{~Hz})$ and D1 $(90-180 \mathrm{~Hz})$ that would produce $\mathrm{WE}=0$ since there was no significant information in subband D1. Decomposition level 2 would produce subband A2 $(0-45 \mathrm{~Hz}), \mathrm{D} 2(45-90 \mathrm{~Hz})$, and D1. The WE value would increase because some information was lied in $\mathrm{A} 2$ and $\mathrm{D} 2$ that make the information spread more even. Higher decomposition level would produce subbands with a more even distribution of information so that WE value will be higher [12].

The proposed method can produce accuracy using only five features. This shows that the proposed method is good enough to separate normal PVC and ECG. The MWE method is affected by the selection of the mother wavelet and decomposition level. Exploration of mother wavelets and the right level of decomposition to get higher accuracy can be the next research topic.

In previous studies, deep learning was used to detect 17 classes of cardiac arrhythmia [13]. The extraction method used is high order statistics. Meanwhile, in other studies, several classifiers such as SVM, NN, DT with KNN and PCA and ICA were used as feature reduction methods [5]. Both studies resulted in an accuracy of up to 99\%. The use of 10 PCs on PCA with Fuzzy C-mean results in an accuracy of $80.91 \%$ [14]. Almost the same method with WE was shown in [15]. In this study multilevel wavelet packet entropy (MWPE) was used for lung sound feature extraction. If MWE was based on DWT, then MWPE was based on wavelet packet decomposition (WPD). The results of the study showed that MWPE level 4 produced the highest accuracy for the classification of five pulmonary sound classes. Some studies produce higher accuracy compared to this study while some studies have resulted in lower accuracy. This shows that the proposed method is quite competitive regarding accuracy. It is still possible to increase accuracy in this method by selecting the right parameters.

\section{Conclusion}

This study describes the classification of PVC based on ECG signals using MWE and SVM. MWE decomposes the ECG signal using several decomposition levels then calculates WE and makes it as feature. SVM is responsible for classifying the characteristics that become the input. The resulting accuracy is a maximum of $94.9 \%$ using quadratic SVM, DB2 with level 5 and 5fold CV. This result is quite competitive compared to other studies considering that with a simple method, few features can produce accuracy up to $94.9 \%$. This method was influenced by decomposition level and mother wavelet. In this study, all the decomposition result sub-bands were used for WE calculation. The optimal selection of subband for WE calculation can be an advanced research topic.

\section{References}

[1] Brosche TAM, The EKG Handbook. Jones \& Bartlett Publisher, (2010).

[2] Ashley EA, \& Niebauer J, "Conquering the ECG - Cardiology Explained - NCBI Bookshelf," in Cardiology Explained, Remedica, (2004).

[3] Lanatá A, Valenza G, Mancuso C, \& Scilingo EP, "Robust multiple cardiac arrhythmia detection through bispectrum analysis," Expert Syst. Appl., vol. 38, no. 6, pp. 6798-6804, (2011).

[4] Mitra M, \& Samanta RK, "Cardiac Arrhythmia Classification Using Neural Networks with Selected Features," Procedia Technol., vol. 10, pp. 76-84, (2013).

[5] Kaya Y, \& Pehlivan H, "Classification of Premature Ventricular Contraction in ECG," Int. J. Adv. Comput. Sci. Appl., vol. 6, no. 7, pp. 34-40, (2015).

[6] Rosso OA et al., "Wavelet entropy : a new tool for analysis of short duration brain electrical signals," J. Neurosci. Methods, vol. 105, pp. 65-75, (2001)

[7] Physionet.org, "ECG Database." [Online]. Available: http://physionet.org/physiobank/database/\#ecg.

[8] Rizal A, \& Hadiyoso S, "ECG signal classification using Hjorth Descriptor," 2015 International Conference on Automation, Cognitive Science, Optics, Micro Electro-Mechanical System, and Information Technology (ICACOMIT), 2015, no. 2, pp. 87-90., (2015)

[9] Xu A, Ye Q, Qiang J, Shan S, \& Zhang D, “A wavelet time entropy algorithm for ultrasonic signal detection," Comput. Model. New Technol., vol. 18, no. 12, pp. 69-73, (2014).

[10] Cortes C, \& Vapnik V, "Support-vector networks," Mach. Learn., vol. 20, no. 3, pp. 273-297, (1995).

[11] Palaniappan R, Biological Signal Analysis. Ventus Publishing ApS, (2010).

[12] Safara S, Doraisamy S, Azman A, Jantan A, \& Ranga S, "Wavelet packet entropy for heart murmurs classification," $A d v$. Bioinformatics, vol. 2012, (2012).

[13] Yildırım O, Pławiak O, Tan TS, \& Acharya UR, "Arrhythmia detection using deep convolutional neural network with long duration ECG signals," Comput. Biol. Med., no. September, (2018).

[14] Jenny NZN, Faust O, \& Yu W, “Automated Classification of Normal and Premature Ventricular Contractions in Electrocardiogram Signals," J. Med. Imaging Heal. Informatics, vol. 4, no. 6, pp. 886892, (2014).

[15] Rizal A, Hidayat R, \& Nugroho HA, "Multilevel wavelet packet entropy: A new strategy for lung sound feature extraction based on wavelet entropy," 2017 International Conference on Robotics, Automation and Sciences (ICORAS), pp. 1-5., (2017) 